\title{
DIGITAL CUSTOM DENTURE DESIGN WITH NEW ABRASIVE COMPUTER TOMOGRAPHY AND RAPID PROTOTYPING TECHNOLOGIES
}

\author{
C. C. CHANG', M. Y. LeE $^{2}$ AND Y.C. $\mathrm{KU}^{3}$ \\ ${ }^{1}$ Department of Mechanical Engineering, Kun-Shan University of Technology, Tainan \\ ${ }^{2}$ Department of Mechanical Engineering, Chang Gung University, Taoyuan \\ ${ }^{3}$ Department of Prosthetic Dentistry, Chang Gung Memorial Hospital, Taoyuan, Taiwan
}

\section{ABSTRACT}

The fixed partial denture produced by conventional method is greatly relied on the skill and experience of dental technician. Its quality and accuracy depends mostly on the technician's subjective judgment. In addition, the manual process involves many procedures that require a long time to complete. Most important, it does not preserve any quantitative information for future retrieval.

In this article, a new device for scanning denture images and reconstructing $3 D$ digital information of dental model by abrasive computer tomography (ACT) was designed in-house and proposed. The fixed partial denture was then produced by the rapid prototyping $(R P)$ and computer numerical control (CNC) machining methods based on ACT scanned digital information. A force feedback sculptor (FreeForm system, Sensible Technologies Inc., USA) accompany with 3D Touch technology was applied to modify the morphology design of denture. It enables the dentist to perform digital manipulation of denture profile with real-time and user-friendly human interactive operation without relying on CAD/CAM technician or dental technician. In this article, the comparison of conventional manual operation and digital manufacture using both RP and CNC machining technologies for denture production was presented. In addition, a digital custom denture manufacturing protocol integrating proposed computer abrasive teeth profile scanning, computeraided denture design, 3D touchable force feedback feature modification and advanced denture manufacturing techniques were suggested. These proposed methods provide solid evidence that digital design and manufacturing technologies may become a new avenue for custom-made denture design, analysis, and production in 21 th century.

Biomed Eng Appl Basis Comm, 2003 (June); 15: 115-123.

Keywords: digital custom denture design, $\mathrm{CNC}$ machining, rapid prototyping, Freeform system, abrasive computer tomography

\section{INTRODUCTION}

Received: June 9, 2003; Accepted: June 18, 2003

Correspondence: M. Y. Lee, Professor

Department of Mechanical Engineering, Chang Gung

University, 259 Wen-hwa lst road,

Taoyuan 333, Taiwan

Email: leemiy@mail.cgu.edu.tw
According to the American Dental Association, approximately 113 million American adults are missing at least one tooth, and 19 million have no teeth at all. Many of these people use removable dentures or fixed partial dentures to restore their missing teeth. But removable dentures can be uncomfortable and troublesome, and they often provide a patient with only a fraction of the chewing force of natural dentition. Fixed prostheses are more effective than removable 
dentures but require grinding healthy adjacent healthy teeth to stubs before fabrication new prostheses. The current manufacturing of removable denture, crowns, or bridges is a labor-intensive work that undergoes substantial processing, with the result that a lot of information is lost and the process takes a long time to complete [1].

The integrated technologies of CAD/CAM and RP are initially applied in automotive and aerospace industries. These techniques are able to make complicated, customer-specific product immediately, without involving time-consuming intermediate stages such as the manufacture of dental moulds [2-3]. Recently, many dentists begin to use these technologies to design and fabricate dental restorations. There are several commercial available CAD/CAM systems (such as Duret, DEntiCAD, CEREC, etc.) were adopted by dentists. Duret et al. obtained dental data by laser scan and produce the prosthesis through a milling machine [4-5]. DentiCAD was developed by Rekow in 1989 [6-7]. A micro mechanical probe is used to obtain the teeth profile information, and the abutment of crown is in tolerance of $40-60$. The CEREC system consisted of image capture, prosthesis design and milling processes, is developed by SIEMENS Ltd. The image is capture by a CCD camera, and prosthesis is produced by 3 -axis milling machine. [8-10].

Computerized digital design and manufacturing procedure usually starting from reverse engineering (RE) operation. The RE technique has widely applied in many aspects, such as industrial manufacturing, automotive and aerospace industry, fabrication and design of arts, etc. Different methodologies of RE are used to produce a computer-aided design (CAD) model from the digitization of a given object by using a coordinates measuring machine (CMM) [11-13]. The CMM system can be divided into two types, the contact system and non-contact system. The contact CMM can automatically generate digitized surface data from a physical model by means of a touch probe. Generally, a touch probe is slow in acquiring points and the digitization process is very time-consuming. The radius compensation of the probe has to be considered in capturing data. A touch probe also limits the quality of measuring in a complex surface.

Another approach is adopted by the non-contact digitization of surfaces on optical techniques, which are much more efficient in measuring speed and reduce the human labor. Because of non-contact CMM, no probe compensation is needed and the mass data can be quickly obtained in a short time. A number of systems based on optical methods have been developed and commercized. For example, laser scanners have a very high data rate (up to $2500 \sim 3500$ points per second) and good resolution (the order of 5-10 $\mu \mathrm{m}$ )
[14-15]. But, laser scanners are so sensitive to the surroundings of light that the accuracy of measurement is easily affected. Another vision systems have lower resolution (about the order of 10-100 $\mu \mathrm{m}$ ); however, they can acquire millions of data points simultaneously over a large spatial range without moving the optical head [16-17]. A wide range of RE applications was utilized abovementioned digitization techniques [1819]. For example. The integration of a 3D vision sensor and a CMM is applied to perform the reverse engineering of freeform surfaces [20].

However, there are still limitations of the contact or non-contact types of CMM in measuring complicated objects, which have the under-cut or inner-structure [2-3]. Although the X-ray microfocus computer tomography (CTM) and magnetic resonance imaging (MRI) are able to overcome above limitations, they are quite expensive or harmful to human bodies, and need a well-trained technician to operate the system. Therefore, a new device uses the abrasive method to remove the inlaid object layer by layer and capture the cross-sectional image of each layer with a CCD camera was proposed [28]. We named the device as abrasive computer tomography (ACT) apparatus. In this article, digital custom denture design and manufacturing is proposed here by integrating proposed abrasive computer tomography, CNC machining and rapid prototyping technologies.

\section{DIGITAL DENTURE MANU- FACTURING}

Figure 1 shows the flowchart of proposed integrated digital custom denture design and manufacturing process. It is noted that various techniques such as ACT teeth profile image capture, 3D computer-aided denture design and modification with 3D touch technology, and digital manufacturing (i.e. CNC machining and RP casting) were utilized in

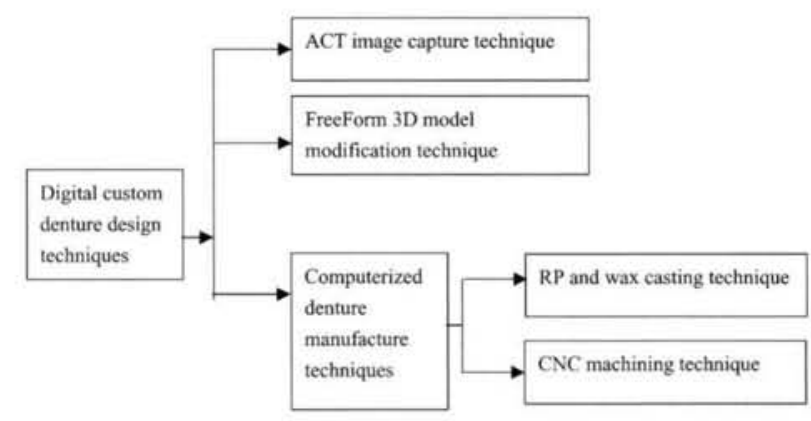

Fig 1. The integrated techniques of digital custom denture design techniques 
the study. First, an ACT apparatus was designed to capture the image of the sliced plaster dental model and to reconstruct 3D CAD model from digital image data. Once the 3D CAD denture model was built, two different computerized digital manufacturing techniques (i.e. CNC machining and Rapid wax prototyping) were used for physical denture production. The $3 \mathrm{D}$ touch technology was also used to help dentist to modify computerized denture model.

\section{ACT IMAGE CAPTURE TECH- NIQUE}

\subsection{Hardware Design of ACT}

Figure $2 \mathrm{a}$ and $2 \mathrm{~b}$ show the design configuration and picture of in-house made ACT apparatus. The original design concept of ACT apparatus is to design a simple device with scanning function of commercial $\mathrm{X}$-ray CT scanner. The unique feature of this ACT apparatus is capable to slice the inlaid physical dental plaster model layer by layer abrasively and in the same time capturing the cross-sectional image of each layer by a CCD camera (1.67 million pixels, Pixera Corporation, USA). The captured images are then transmitted to a computer for image processing and 3D reconstruction. Figure $2 \mathrm{c}$ shows the physical dental plaster model was embedded in silicone or gypsum material and sliced layer by layer through the rolling

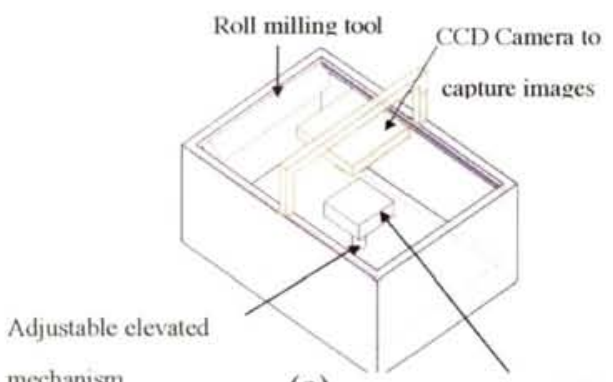

mechanism

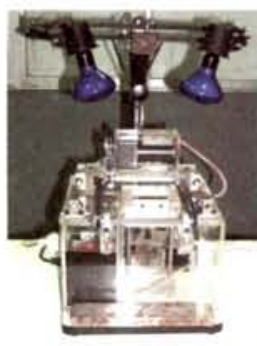

(b)
Inlaid objects

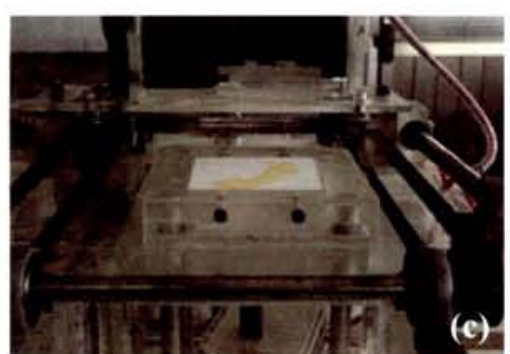

Figure 2. (a) Concept design of abrasive computed Tomography; (b) Apparatus of abrasive computed Tomography; (c) Slicing teeth plaster model embedded in silicon or gypsum tool. The scanning workspace of the ACT apparatus is $80 \times 80 \mathrm{~mm}$. The dimensional precision of the ACT apparatus is $0.063 \mathrm{~mm}$. The precision can be enhanced, if the higher quality of CCD camera is used.

\subsection{Image Binary Segmentation and 3D Reconstruction}

\subsubsection{The Processing of Binary Segmentation}

The binary segmentation of image processing is to threshold the gray gradient of the image under the control of the threshold value. Mathematic algorithms to threshold the gray gradient of images are described as follows:

For a second order of threshold function:

$$
f(x, y)= \begin{cases}1 & f(x, y) \geq T \\ 0 & f(x, y)<T\end{cases}
$$

For n-order of threshold function:

$$
f(x, y)=\left\{\begin{array}{lr}
N_{1} & f(x, y) \geq T_{1} \\
N_{2} & T_{2} \leq f(x, y)<T_{1} \\
\vdots & \vdots \\
N_{n} & T_{n} \leq f(x, y)<T_{n-1}
\end{array}\right.
$$

Where $f(x, y)$ is the value of gray gradient of pixel and $T$ is threshold value.

In the second order of binary segmentation, the pixel in the image will be kept when the value of gray gradient is larger than the threshold valve, otherwise it will be treated as a void. For n-order of binary segmentation, the pixel will be kept when the value of gray gradient is in the range of threshold value. Figure 3a shows the image of the sliced plaster dental model captured by ACT apparatus. After the binary

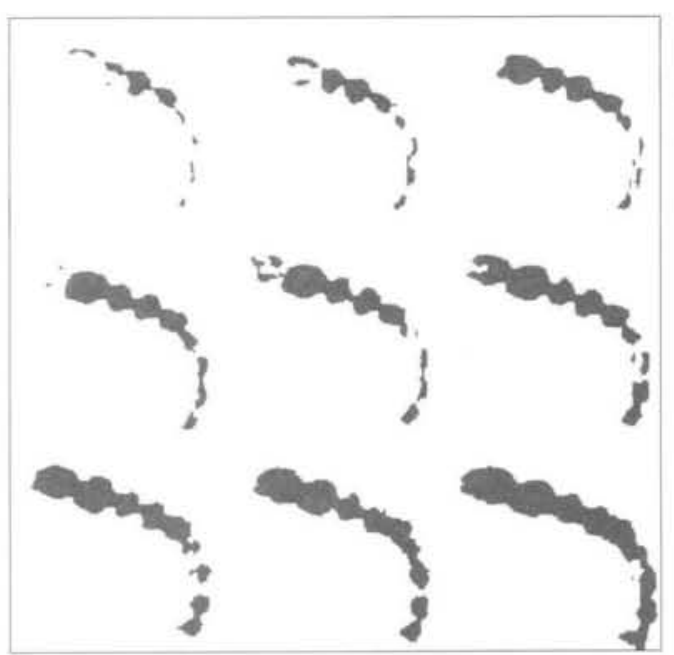

Fig 3a. Images of the sliced plaster teeth model by ACT apparatus. 
segmentation of image processing, a cross-sectional dental image can be obtained in Figure $3 \mathrm{~b}$. The boundaries of images for each slicing layer are shown in Figure 3c.

\subsubsection{Reconstruction 3D CAD Model}

There are several methods to rebuild 3D CAD model during image processing, such as stack region growing, quad tree split etc. In this paper, different approach to reconstruct 3D CAD model was proposed and described as follows:

1. To stack point clouds of all boundaries as shown in Fig. 4a.

2. To generate Streolithography (STL) format of point clouds as shown in Fig. 4b.

3. To rebuild 3D CAD Model of teeth model as shown in Fig. 4c.

In order to implement above reconstruction procedures, several commercial software such as LabView, CopyCAT (Delcam International, Birmingham, UK), and PowerSHAPE (Delcam International, Birmingham, $\mathrm{UK}$ ) were used to develop the interface software.

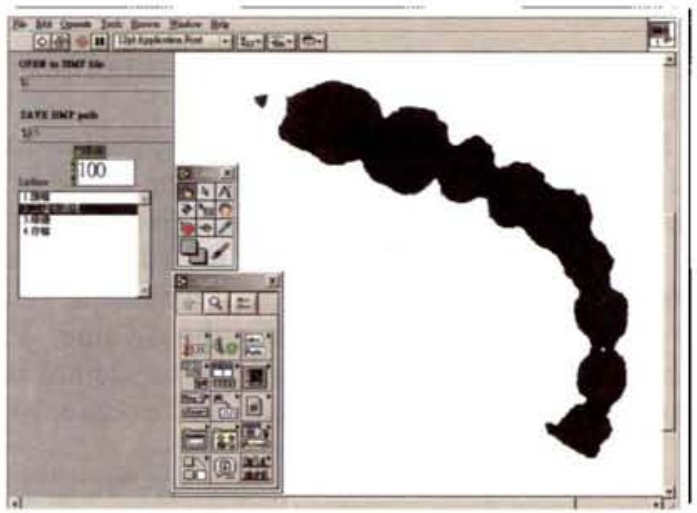

Fig 3b. A cross-sectional teeth image after binary segmentation

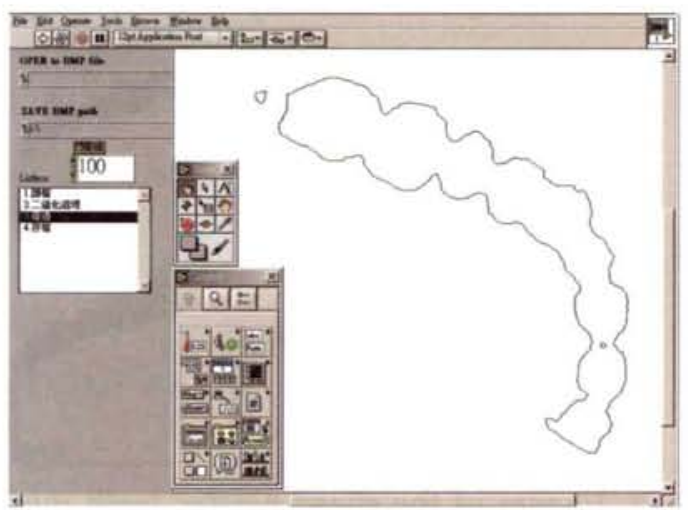

Fig 3c. A cross-sectional boundary of teeth model
It is noted that many objects with complicated geometries such as the under-cut or inner structure are difficult to scan using conventional CMM machine. Unfortunately, in many medical applications the subject exhibits complex geometries, such as the artificial teeth, bone prosthesis, auricular prosthesis, craniofacial implant etc. Traditionally, CT or MRI machine is utilized to scan objects in medical applications. However, those facilities are harmful to human because of X-ray radiation. In addition, CT and MRI machine are usually available only in wellequipped hospitals, medical center or clinical research institutions. Also, those expensive machines usually require well-trained technician to operate. In this research, the ACT apparatus was used to obtained sectional images of physical models in medical applications. The format of the captured images is Bitmap. The boundaries of each layer are generated with binary segmentation and numerical schemes. Finally, 3D CAD model can be reconstructed as shown in Figure 4.

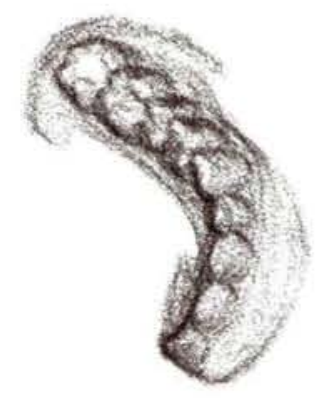

(a)

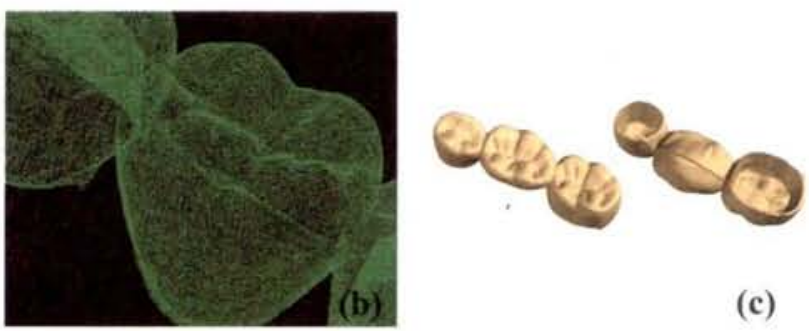

Fig 4 (a) Point clouds of teeth model composed by all layers. (b) STL format of teeth model. (c) 3D CAD of teeth model by shading.

\section{FREEFORM SYSTEM OF 3D MODEL MODIFICATION TECH- NIQUE}

Freeform System (SensAble Technologies, Inc) is a 3D touch technology that allows users to interact with digital objects in the computer just like they do in the real world. Modelers sculpt as naturally in digital 
form as they do in clay and surgeons perform their crafts with virtual patients instead of actual people. The technology makes working in three dimensional computer interactions more natural, efficient and intuitive by allowing users to have continuous, twoway interaction with their work. It provides the ability to directly manipulate models and data, which mean less time is spent setting parameters. Direct manipulation metaphors can also considerably reduce the learning curve required for $3 \mathrm{D}$ medical applications.

The digital denture data (STL format) can be easily modified by freeform as shown in Figure 5. Dentists can directly access and modify without relying on $\mathrm{CAD} / \mathrm{CAM}$ technician or laboratory technician. The flow chart of freeform 3D touch technology is shown in Figure 6. The PHANTOM hardware (Sensible Technologies Inc., USA) provides precision positioning input and high fidelity, forcefeedback output. GHOST software (Sensible Technologies Inc., USA) works as the engine, handling complex computations and allowing developers to deal with simple, high-level objects and physical properties such as location, mass, friction and stiffness.

\subsection{CNC Machining Technique}

Once the 3D CAD model was reconstructed and modified, the tool path can be generated and transmitted into 4-axis CNC milling machine to produce the prosthesis. There are two different ways to generating the tool path, one is for above three teeth denture and another is for single-teeth prostheses. Four tool paths are produced to mill the above three teeth denture since the bend force is too large to stand cutting, while one integrated tool path is directly applied in machining a crown (single unit prostheses). The flow chart is shown in Figure 7.

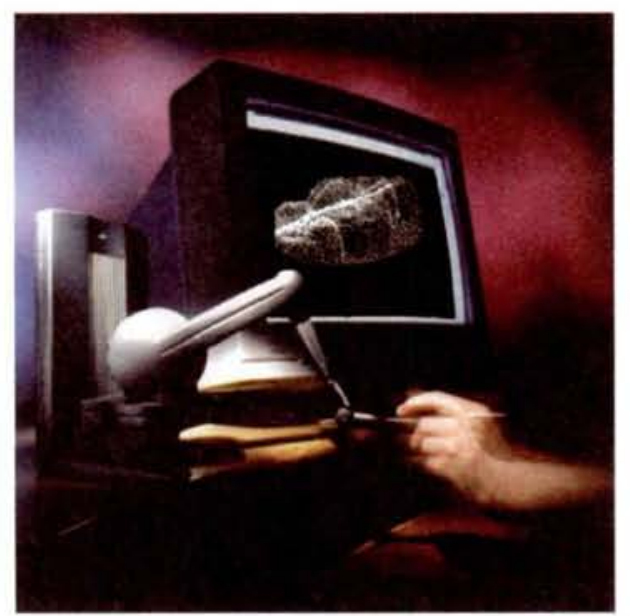

Fig 5. The digital denture data (STL format) modified by FreeForm

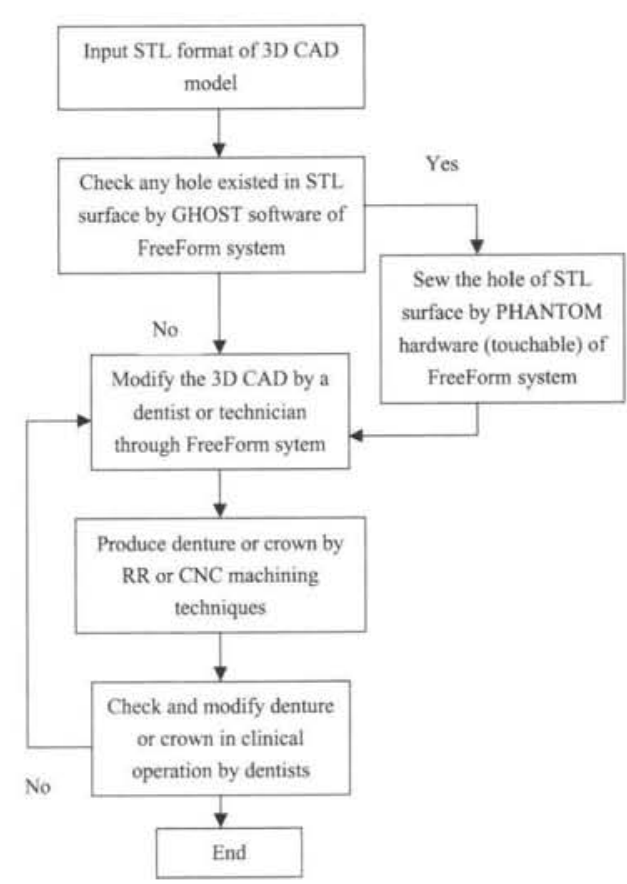

Fig 6. The flow chart of digital denture data modified by 3D touch technology of FreeForm system

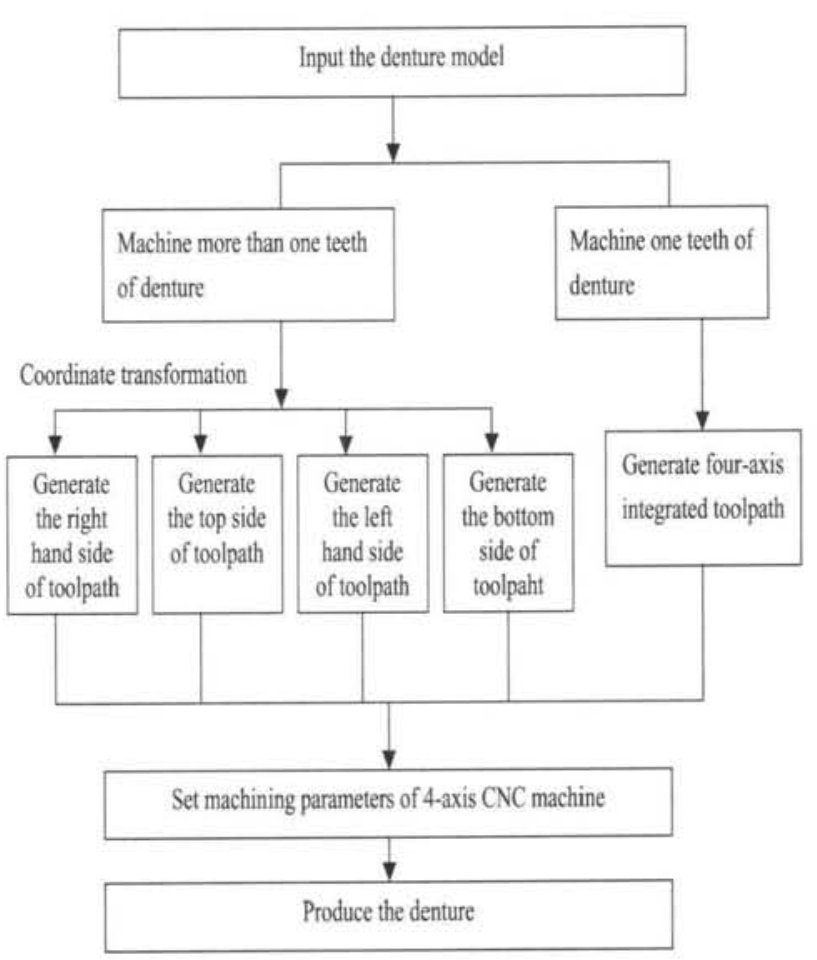

Fig 7. The flow chart of $\mathrm{CNC}$ machining the denture 


\subsection{CNC User Interface Module}

The CNC user interface module is developed using Visual Basic (VB) language in PowerMILL (Delcam International, Birmingham, UK) environment. The macro commands are specifically assembled for machining denture. The input user-interface window including data entry for the type of tool, material size and brand of controller. The output user-interface window is for generating tool paths. In Figure 8, the gererated tool paths simulating the real machining conditions was demonstrated.

\subsection{CNC Denture Manufacturing}

As mentioned earlier, single integrated tool path to mill one-tooth prostheses is demonstrated in Figure 9a, while four different tool paths to mill a three-teeth prosthesis are shown in Figure 9b. In addition, one titanium crown is machined by a 4-axis CNC milling machine as shown in the Figure 10a and 10b,

\section{RP AND WAX CASTING TECH- NIQUE}

The RP is a new layer-by-layer forming process and belongs to the material addition manufacturing process. It can rapidly produce products without any mold. Different kinds of rapid prototyping machine (RPM) were developed by using different mechanisms or materials, such as sterolithography apparatus (SLA), laminated object manufacturing (LOM), selective laser sintering (SLS), fused deposition modeling (FDM), and Multi-jet modeling (MJM) [22-24]. Rapid prototyping casting patterns for implant crowns are used as patterns to cast the parts in gold or dental alloy [1]. In this research, the STL format of dental model was created for rapid prototyping as shown in Figure 11. The wax pattern of teeth is produced by Actua 2100 (3D system Inc., USA), and can be cast into metal prosthesis for clinical cure. The final artificial teeth produced using RP technique as shown in Figure 12.

\section{DISCUSSION}

In this article, abrasive computer tomography method and new ACT device is proposed to quickly capture the complex geometric characteristics of teeth. The advantages of this approach are:

a. ACT apparatus is a more convenient and economic way to obtain the images of CT for complicate objects, with under-cut or inner structure.

b. All the geometric data is saved in the form of digital format, which can be easily manipulated during design and shape modification process.

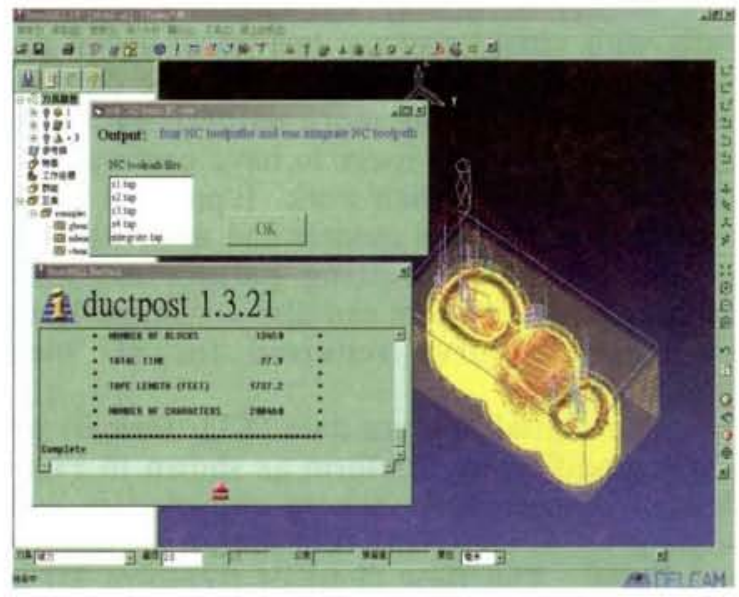

Fig 8. Tooth path generation and simulation

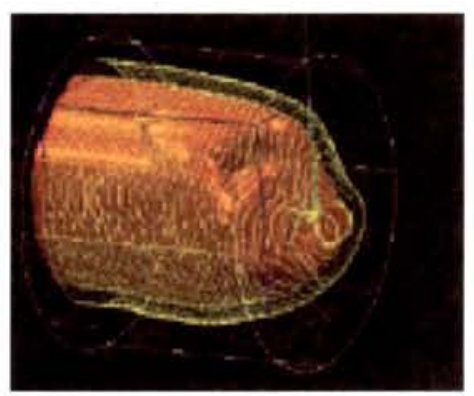

(a)

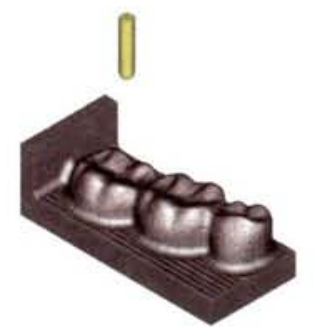

Top side

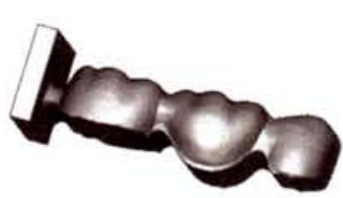

Right hand side

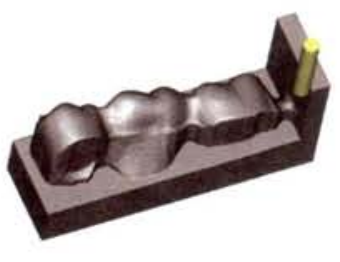

Left hand side

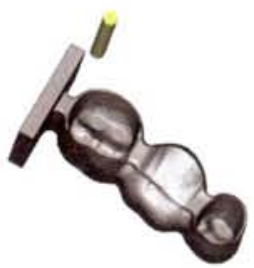

Bottom side

(b)

Fig 9. (a) One integrated toolpath of one teeth, (b) Four different toolpaths of three teeth 


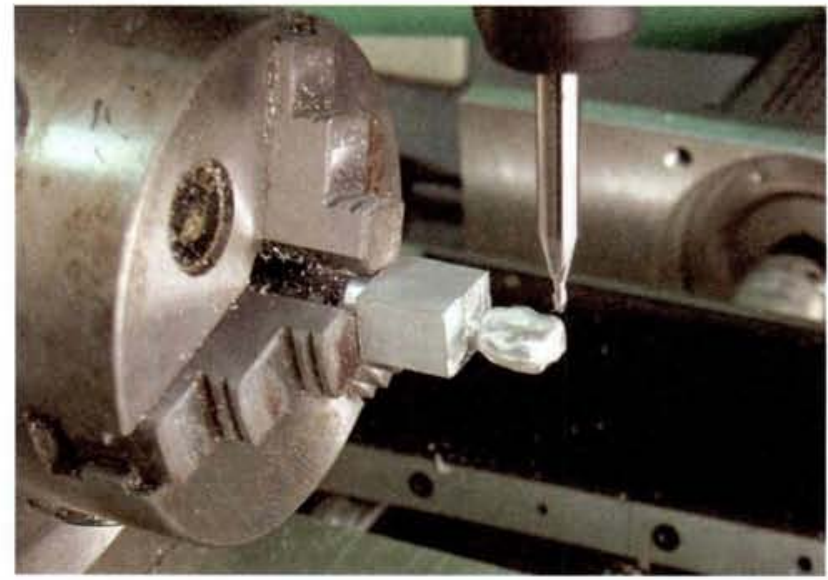

(a)
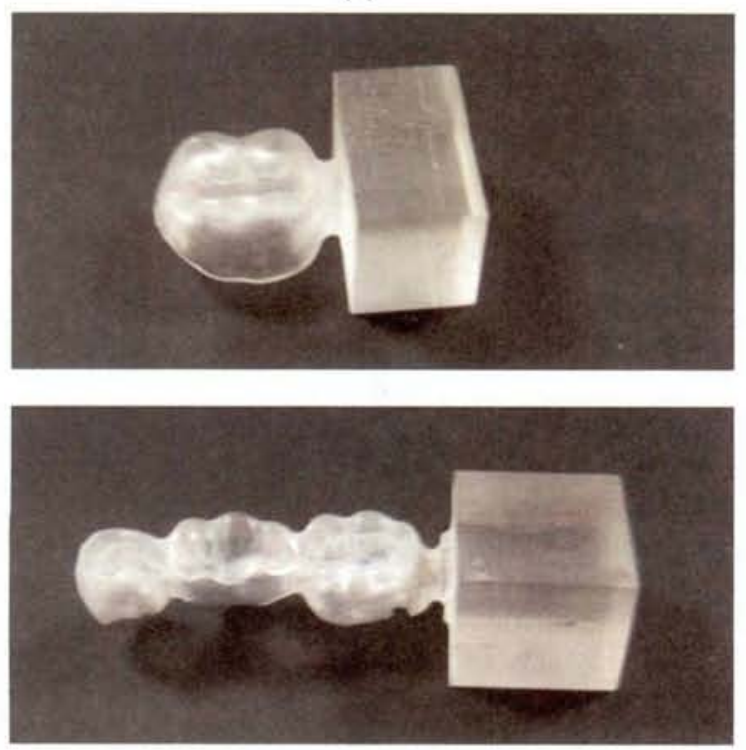

(b)

Fig 10. (a) One crown (Titanium) machining by 4axis CNC milling machine, (b) Examples after CNC machining

Once the subject lost his/her false teeth (denture), the digital data file can be quickly retrieved and a replacement can be produced without go through tedious conventional denture fabrication procedures.

c. The images from ACT can be quickly converted to STL format for rapid prototyping, or reconstructed to a $3 \mathrm{D}$ CAD model for $\mathrm{CNC}$ machining.

d. In medical applications, ACT may be useful to provide 3D CAD models for surgical rehearsal, and produce all kinds of prosthesis through the techniques of RP/RT.
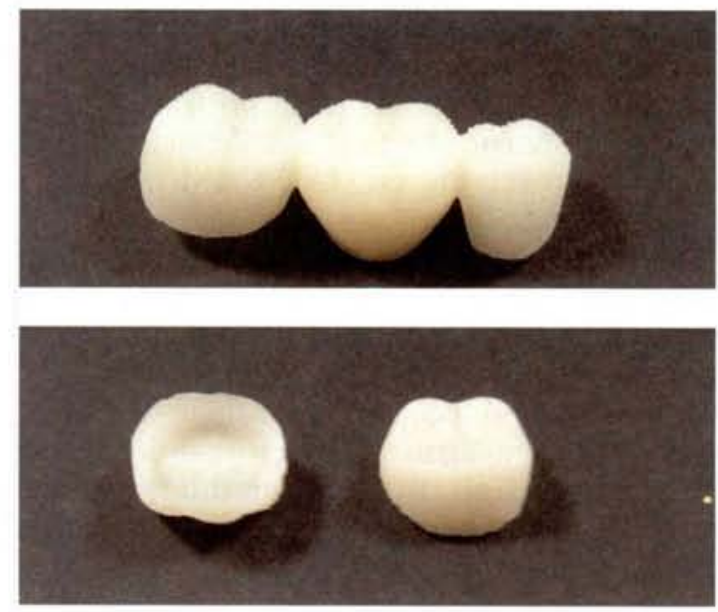

Fig 11. Rapid prototyping of teeth model by Actua 2100 (3D System Inc.)
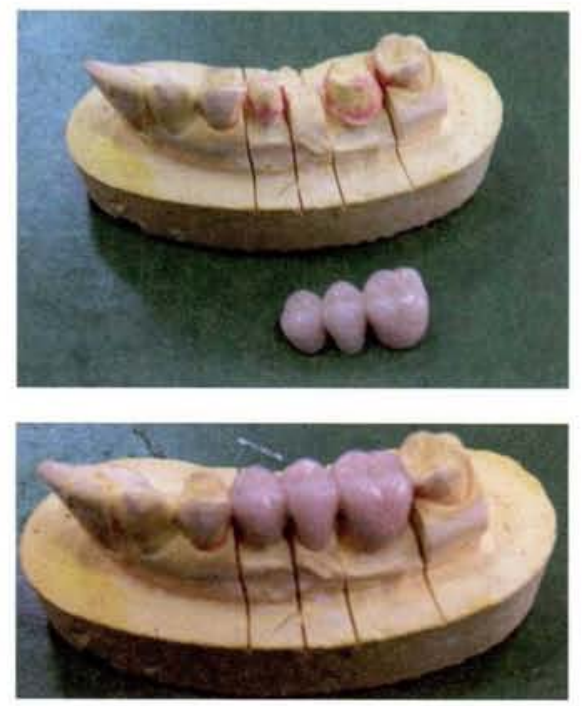

Fig 12. Artificial teeth fabricated by means of ACT techniques

e. Currently, fingerprints and eyeball characteristics are used for personal identification in case of accident. However, the finger and eyeball are soft tissues, which can be easily destroyed by fire. Therefore, the digital teeth information can be very useful in personal identification.

An integrated computer abrasive scanning, digital denture design, and numerical denture manufacturing was proposed in this paper. Several denture design cases using proposed approach provide solid evidence that digital information and manufacturing technologies may become a new avenue for denture 
design, analysis, and production in the next century. A force feedback Freeform device was suggested used for modification of digital teeth profile. Due to its user-friendly human interface, the dentist can directly access without relying on $\mathrm{CAD} / \mathrm{CAM}$ technician or dental technician.

In addition, the proposed digital denture manufacturing method using CAD/CAM technologies demonstrated the feasibility for producing accurate false teeth in this paper. The digital teeth data can further used in mesh generation for finite-element CAE analysis. The comparison between traditional manual operation and digital design methods forcustom denture design and manufacturing was summarized in Figure 13.

\section{CONCLUSION}

Digital denture manufacturing, a combination of digital imaging, CAD/CAM, and RP technologies, was proposed for denture manufacturing. A newly developed ACT apparatus is proposed for denture image scanning and reverse engineering. The advantages of ACT were found as follows.

1. ACT apparatus is a convenient and an economic way to obtain the images of CT for

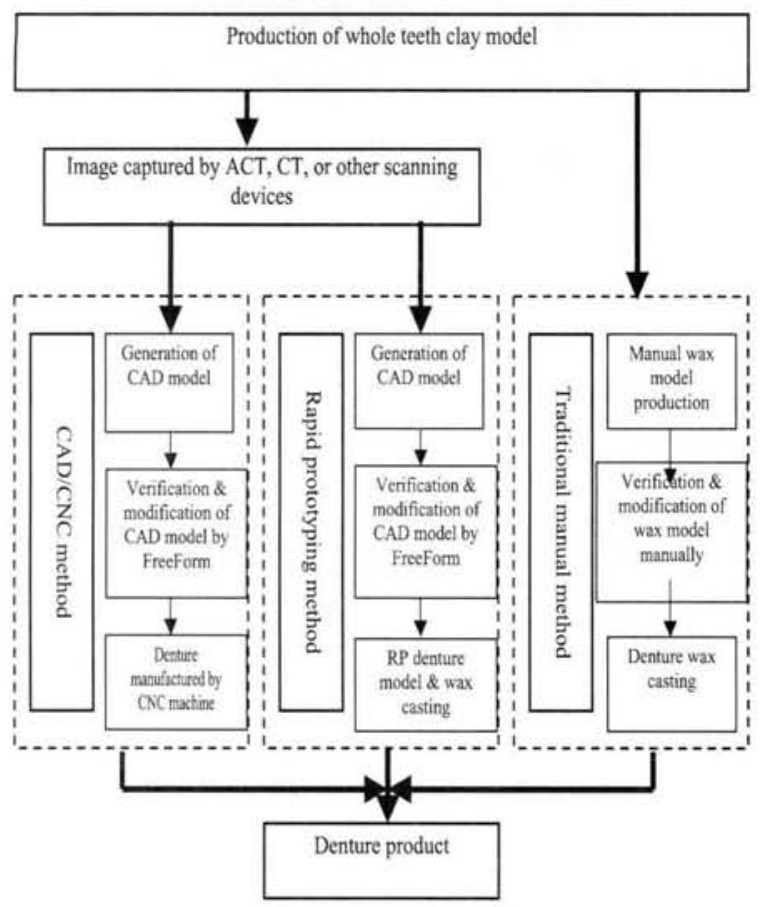

Fig 13. Comparison of traditional manual operation and digital custom denture design methods complicated objects with existed under-cut or inner structure.

2. The images from ACT can be quickly converted to STL format for rapid prototyping fabrication and 3D CAD model reconstruction for $\mathrm{CNC}$ machining.

3. ACT may apply to different clinical applications to provide 3D Computer models for surgical planning, operation simulation, and custom implant or prosthesis design using RP/RT techniques.

In this article, the digital custom denture design and manufacturing was demonstrated. By using technologies of CAD/CAM and RP, it is able to make complicated, customer-specific product immediately, without involving time-consuming intermediate stages such as the manufacture of dental moulds. The proposed digital denture manufacturing technique may become a highly promising production process for dental prostheses such as crowns and bridges in the future.

\section{REFERENCES}

1. Schmitt S, "Rapid prototyping custom dental implants", Rapid Prototyping Report, February 2001.

2. Chang $\mathrm{CC}$ and Chiang HW, "Reconstruction the CAD model of complex object by abrasive computed tomography", 2002 IEEE/ASME International Conference on Advanced Manufacturing Technologies and Education in the 21st Century, Taiwan, 2002.

3. Chang CC and Chiang HW, "Three-Dimensional Image Reconstruction of Complex Objects by Abrasive Computed Tomography Apparatus", accepted by International Journal of Advanced Manufacturing Technology, 2002.

4. Duret F, Blouin JL. and Duret B, "CAD/CAM in dentistry", JAM Dent Assoc., 117, pp.715-720, 1988.

5. Duret F. et al., "Method of Making a Prosthesis, Especially a Dental Prosthesis", Technical report, US Patent Nr. 4742464, 1988.

6. Rekow DE, "The Minnesota CAD/CAM System DentiCAD", Technical report, U. of Minnesota, 1989.

7. Rekow DE, "Method and apparatus for modeling a dental prosthesis", Technical report, US patent Nr. $5273429,1993$.

8. Calamia JR, "Advances in computer-aided design and computer-aided manufacture technolody", Current Opinion in Cosmetic Dentistry, pp. 67-73, 1994. 
9. Tinschert J, Zwez D, Marx R, Anusavice KJ, "Structural reliability of alumina-, feldspar-, leucite-, mica- and zirconia-based ceramics", J Dent, 28(7), pp. 529-535, 2000.

10. Apholt W, Bindl A, Luthy H, Mormann WH, "Flexural strength of Cerec 2 machined and jointed InCeram-Alumina and InCeram-Zirconia bars", Dent Mater, 17(3), pp. 260-267, 2001.

11. Varady $T$, Martin RR, and Cox J, "Reverse engineering of geometric models-an introduction", Computer-Aided Design, 29(4), pp. 255-268, 1997.

12. Yau HT, "Reverse engineering of engine intake ports by digitization and surface approximation," International Journal of Machine Tools and Manufacture, 37(6), pp. 855-871, 1997.

13. Motovalli S, "Review of reverse engineering approaches," Computers and industrial Engineering, 35, pp. 25-28, 1998.

14. Chan V, Bradley C and Vickers GW, "Automating laser scanning of 3-D surfaces for reverse engineering", SPIE, 3204, pp. 156-164, 1997.

15. Bradley C, Milroy M. and Vickers GW, "Reverse engineering of quadric surfaces employing 3D laser scanning", Proceedings of the Institution of Mechanical Engineers, Part b., 208, pp.21-28, 1994.

16. Niem $\mathrm{W}$ and Wingbermuhle $\mathrm{J}$, "Automatic reconstruction of $3 \mathrm{D}$ objects using a mobile monoscopic camera", Proceedings of International Conference on Recent Advances in 3-D Digital and Modeling, pp. 173-180, 1997.

17. El-Hakim SF. and Pizzi N, "Multicamera visionbased approach to flexible feature measurement for inspection and reverse engineering", Optical Engineering, 32, 1993.

18. Beraldin JA, Coumoyer L, Rioux M, et al. "Object model creation from multiple range images: acquisition, calibration, model building and verification", Proceedings of International Conference on Recent Advances in 3-D Digital and Modeling, pp. 326-333, 1997.

19. Soucy M, Godin G, Braibeau R, et al. "Sensors and algorithms for the construction of digital 3-D colour model of real object", Proceeding of International Conference on Image Processing, 2, pp. $409-412,1996$.
20. Carbone V, Carocci M, Savio E, et al. "Combination of vision system and a coordinate measuring machine for the reverse engineering of freeform surfaces," International Journal of Advance Manufacturing Technology, 17, pp.263$171,2001$.

21. Lee MY, Chang CC and Lin CC, "3D Image reconstruction and rapid prototyping models improve defect evaluation, treatment planning, implant design, and surgeon accuracy", IEEE Engineering in Medicine and Biology, Vol. 21, No. 2, pp. 38-44, 2002.

22. Onuh O, Yusuf YY, "Rapid prototyping technology: applications and benefits for rapid product development," Journal of Intelligent Manufacturing, 10, pp-301-311, 1999.

23. Kai CC , Lim CS, Leong KF, "Rapid Prototyping" GPrinciples \& Applications in Manufacturing," World Scientific Pub. Co., November 2002.

24. Pham DT, Gault RS, "A comparison of rapid prototyping technologies," International Journal of Machine Tools \& Manufacture, 38 pp 1257-1287, 1998.

25. Kai CC, Chou SM, Lin CS, et al. "Rapid Prototyping Assisted Surgery Planning," The International Journal of Advanced Manufacturing Technology, 14, pp. 624-630, 1998.

26. Altschuler BR, Toboada J and Segreto VA, "IntraOral Dental Laser Grid Surface Mapping", Applications of Optical Instrumentation in Medicine IV. SPIE 70, pp.347-350, 1975.

27. Wang J, Li X, Shaw LL, Harris L, "Powder Delivery in Dental Restoration Rapid Prototyping Process", Solid Freeform Fabrication Proceedings, Solid Freef 546-552, 2001.

28. Chang $\mathrm{CC}$, "Three-Dimensional Image Reconstruction of Complex Objects by Abrasive Computed Tomography Apparatus", Taiwan Patient NO. 164809. 